\title{
Vascular endothelial growth factor receptor 2-mediated angiogenesis is essential for gonadotropin-dependent follicle development
}

\author{
Ralf C. Zimmermann, ${ }^{1}$ Tipton Hartman, ${ }^{1}$ Suzanne Kavic, ${ }^{1}$ Samuel A. Pauli, ${ }^{1}$ \\ Peter Bohlen, ${ }^{2}$ Mark V. Sauer, ${ }^{1}$ and Jan Kitajewski ${ }^{1,3}$ \\ ${ }^{1}$ Department of Obstetrics and Gynecology, Division of Reproductive Endocrinology, Columbia University, \\ New York, New York, USA \\ ${ }^{2}$ ImClone Systems, New York, New York, USA \\ ${ }^{3}$ Department of Pathology, Columbia University, New York, New York, USA
}

\begin{abstract}
Gonadotropins induce ovarian follicle growth that is coincident with increased follicular vasculature, suggesting a role of angiogenesis in follicle development. Functional studies performed in nonhuman primates show that administration of substances that inactivate VEGF block the development and function of preovulatory follicles as demonstrated by histological analysis or hormone measurements. Blockage of function of VEGF receptor 2 (VEGFR-2) alters follicular hormone secretion, suggesting that the intraovarian effect of VEGF might be mediated by this receptor. The specific mechanism by which follicular development was blocked in these previous studies remains unclear, however. Here we characterize the intraovarian role of VEGFR-2 activity on follicular development by choosing a model in which active feedback is absent, the prepuberally hypophysectomized mouse. Hypophysectomy prevents advanced follicle growth and maturation; however, follicle development to the preovulatory stage can be stimulated by administration of gonadotropins. We report that exogenously administered gonadotropins are unable to drive follicle development to the preovulatory stage in the presence of antiangiogenic agent, VEGFR-2-neutralizing Ab's. This inhibition of follicular development is caused by arrests to both angiogenesis and antrum formation. We conclude that the intraovarian VEGF/VEGFR-2 pathway is critical for gonadotropin-dependent angiogenesis and follicular development.
\end{abstract}

J. Clin. Invest. 112:659-669 (2003). doi:10.1172/JCI200318740.

\section{Introduction}

In mammals the de novo appearance and continued increase in vascular networks accompanying progression of follicle development suggests that ovarian function is intimately dependent on follicular angiogenesis. Follicles increase their granulosa cell number and acquire a peripheral theca layer in which new blood vessels form (vasculogenesis) during the transition from a compact, avascular primary to a vascular secondary follicle. Beyond this stage follicular growth becomes gonadotropin dependent and results in the formation of a centrally located, fluid-filled antrum possibly mediated by changes in vascular permeability. Formation of antrum coincides with continued follicular

Received for publication April 24, 2003, and accepted in revised form July 3, 2003.

Address correspondence to: Ralf C. Zimmermann, Department of Obstetrics and Gynecology, Division of Reproductive Endocrinology, College of Physicians and Surgeons, Columbia University, 630 West 168th Street, New York, New York 10032, USA. Phone: (212) 305-8693; Fax: (212) 305-3869;

E-mail:rcz3@columbia.edu.

Conflict of interest: The authors have declared that no conflict of interest exists.

Nonstandard abbreviations used: VEGF receptor 2 (VEGFR-2); hypophysectomized (HX); pregnant mare serum gonadotropin (PMSG); platelet endothelial cell adhesion molecule (PECAM); diaminobenzidine tetrahydrochloride (DAB). angiogenesis resulting in the development of an intricate vascular mesh, which consists of two concentric vascular networks in the peripheral theca layer (angiogenesis) (1). These newly formed ovarian blood vessels likely secure an increasing supply of gonadotropins, growth factors, oxygen, steroid precursors, as well as other substances to the growing follicle (2).

The regulation of follicular angiogenesis is poorly understood (1-7). As suggested from expression analysis $(2,5)$, one of the prime candidates regulating ovarian blood vessel formation is VEGF, also called VEGF-A (8), acting through one of its two receptors or both (8). Further evidence for a role of VEGF in the regulation of gonadotropin-dependent ovarian follicular angiogenesis and folliculogenesis is derived from functional studies. For example, administration of substances that inactivate VEGF block the development and function of preovulatory follicles as demonstrated by analysis of ovarian histology $(2,5)$ or hormone measurements (6). Blockage of the function of the VEGF receptor 2 (VEGFR-2) receptor alters follicular hormone secretion, suggesting that the intraovarian effect of VEGF might be mediated by this receptor (7). The specific mechanism by which follicular development was blocked in these previous studies remains unclear, however. In this study we will specifically investigate whether the VEGF/VEGFR-2 pathway, which is involved in the reg- 
ulation of angiogenesis, survival of endothelial cells, and vascular permeability (8), plays a critical role for gonadotropin-dependent folliculogenesis. In addition, interpretation of results from previous studies $(2,5-7)$ is complicated by the fact that all of them were done in animals with an active feedback mechanism. Because administration of antiangiogenic agents can also influence hormonal feedback loops between the ovary and pituitary gland $(6,7)$, some changes observed in folliculogenesis might be due to this effect. To evaluate the intraovarian role of the VEGFR-2 pathway for gonadotropin-dependent follicle development we chose a model, the prepuberally hypophysectomized (HX) mouse, in which active feedback is absent. In such a model advanced follicle growth and maturation is normally absent; however, follicle development to the preovulatory stage can be stimulated under controlled conditions by the administration of exogenous gonadotropins. Using HX mice, we demonstrate that exogenously administered gonadotropins are unable to drive follicle development to the preovulatory stage in the presence of antiangiogenic agent in form of VEGFR-2-neutralizing Ab's. This inhibition of follicular development is caused by arrest of follicular angiogenesis and a reduction in antrum formation. We conclude that the intraovarian VEGF/VEGFR-2 pathway is critical for gonadotropin-dependent follicle angiogenesis and follicular development.

\section{Methods}

Experimental design. The experiments were designed to investigate the role of VEGFR-2-mediated angiogenesis for gonadotropin-dependent follicle development. We used female CD-1 mice hypophysectomized before day 24 of life (Charles River Laboratories Inc., Wilmington, Massachusetts, USA). Follicle development in such animals arrests at the advanced preantral or early antral stage due to the absence of gonadotropins (9), and mature, preovulatory follicles are generally absent. Advanced preantral follicles are highly responsive to gonadotropins, and the development of mature, antral follicles can be induced by exogenously administered gonadotropins over a 3- to 4-day period (gonadotropindependent growth) (9-11). Absence of pituitary glands in such a model can be verified indirectly by demonstrating (a) insignificant weight gain $(<1.5 \mathrm{~g})$ over a 1 -week period after arrival in the laboratory (12) and (b) vaginal smears typical for a low-estrogenic state. Only animals that met these criteria were included in the study and were randomly assigned to one of four groups 1 week after arrival.

Animals in the stimulation group $(n=10$ per time point) received saline injection $(250 \mu \mathrm{l})$ in the evening of days 8 and 10 after arrival. In the morning of day 9 after arrival, animals received a single dose of $20 \mathrm{IU}$ pregnant mare serum gonadotropins (PMSG) (SigmaAldrich, St. Louis, Missouri, USA) to induce gonadotropin-dependent follicle development. Animals were sacrificed 24 hours (experimental day 1), 48 hours (experimental day 2), and 72 hours (experimental day 3) after PMSG injection corresponding to day 10,11 , or 12 after arrival.

Animals in the treatment group $(n=10$ per time point) received rat anti-VEGFR-2 $\mathrm{Ab}(800 \mu \mathrm{g}$ dissolved in $250 \mu \mathrm{l}$ in the evening of day 8 and 10) (DC 101; ImClone Systems, New York, New York, USA) (13) after arrival. PMSG was administered as described for the stimulation group. Animals were sacrificed at the same time points as in the stimulation group.

Animals in the control group ( $n=10$ per time point) were injected with saline in the evening of day 8 and day 10 ( $250 \mu \mathrm{l}$ of saline), simulating $\mathrm{Ab}$ injection in the treatment group, and in the morning of day $9(200 \mu \mathrm{l}$ of saline), simulating the PMSG injection. Animals were sacrificed at the same time points as in the stimulation group. Inclusion of this group allowed definition of the type and number of follicles developing during the time of experimental intervention in the absence of gonadotropins and antiangiogenic agent.

Animals in the baseline group $(n=10)$ received no intervention and were sacrificed in the evening of day 8 after arrival. This allowed definition of the type and number of follicles present before any intervention was initiated.

One hour before sacrifice all animals were injected intraperitoneally with $1 \mathrm{ml} \mathrm{BrdU}$ reagent (00-0103; Zymed Laboratories Inc., South San Francisco, California, USA) per $100 \mathrm{~g}$ mouse.

Dosage of anti-VEGFR-2 and effect of nonspecific rat IgG. A dose-response curve using anti-VEGFR-2 (DC101) had been performed injecting three groups (three animals per group) at the following dosages twice, 48 hours apart: $0.4 \mathrm{mg}, 0.8 \mathrm{mg}$, and $1.6 \mathrm{mg}$. The effect of DC101 on PMSG-dependent folliculogenesis was evaluated 3 days after injections were initiated. The same level of inhibitory response was achieved with injection of 0.8 $\mathrm{mg}$ or $1.6 \mathrm{mg}$ of Ab. Because there was no difference in the level of inhibition between these two dosages, the 0.8 -mg dose per animal was chosen. In our experiments we used saline instead of nonspecific rat IgG (SigmaAldrich) for sham injections because nonspecific rat IgG increased background staining in immunohistochemistry. Because it is possible that rat IgG could have nonspecific effects on gonadotropin-dependent follicle development, we tested for this possibility by evaluating follicle development induced with PMSG in the presence and absence of nonspecific rat IgG Ab (two dosages of $800 \mu \mathrm{g} 2$ days apart; three animals per group), and no effect of $\mathrm{Ab}$ on follicle development was detected. Therefore, we concluded that sham injections of saline can replace control $\mathrm{Ab}$ injections.

Histology. Following sacrifice, both ovaries and uterus were removed. One ovary and the uterus were fixed in formalin. The second ovary, called the contralateral ovary, was embedded in OCT, flash-frozen in isopentane cooled by dry ice, and stored at $-80^{\circ} \mathrm{C}$. The formalin-fixed organs were weighed after drying with fluid-absorbing paper tissue. Serial $10-\mu \mathrm{m}$ ovarian tissue sections were cut through all formalin-fixed 
a

C

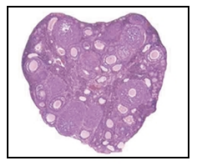

s $5 \sin ^{\circ}$

$\mathrm{T}$

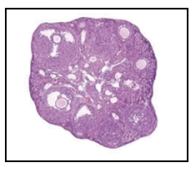

C
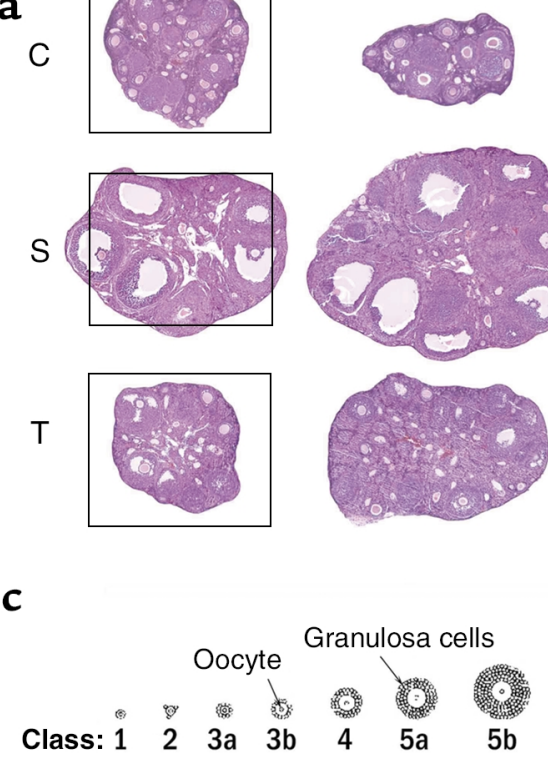
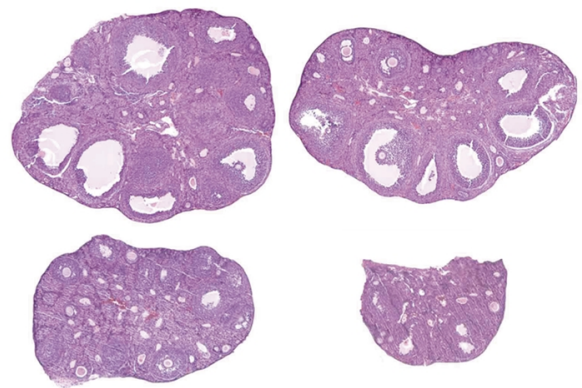

Antrum

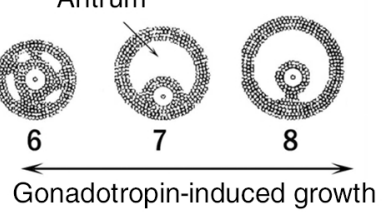

b
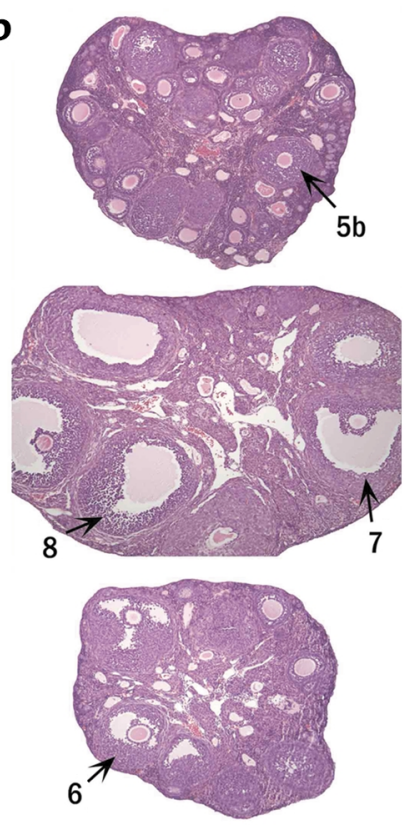

\section{Figure 1}

(a) H\&E staining of mouse ovaries on experimental day 3. Top panel, low-power photomicrographs of three representative whole ovaries from $\mathrm{HX}$ animals not treated with hormone (C, control); no advanced antral stage follicles present. Middle panel, low-power photomicrographs of three representative whole ovaries from HX animals stimulated with PMSG (S, stimulation); at least three advanced antral stage follicles present in each section. Lower panel, low-power photomicrographs of three representative whole ovaries from HX animals treated with PMSG plus anti-VEGFR-2 Ab ( $T$, treatment); early antral stage follicles present in each section. (b) Intermediate-power photomicrographs of a control, stimulation, and treatment ovary (boxed area in a). Numbers indicate classes of follicular development. (c) Follicle classification.

ovaries, and each section was stained with H\&E. Evaluation of serial sections representing whole ovaries allows quantification of the gonadotropin-dependent dynamics of follicle development over time under the different experimental conditions by counting the total number of follicles present in individual ovaries.

Evaluation of individual follicles. To evaluate the effects of experimental interventions at the level of individual follicles, such follicles of interest located in the contralateral ovary from each animal were characterized by (a) examining the development of a microvascular network by staining against PECAM (platelet endothelial cell adhesion molecule) (b) quantifying the number of mitotic granulosa cells staining for BrdU, and (c) determining the presence of proliferating endothelial cells through colocalization of BrdU and PECAM.

Selection of follicles eligible for evaluation. To identify follicles suitable for immunohistochemical or immunofluorescent evaluation fifty $10-\mu \mathrm{m}$ sections from the center of each frozen, contralateral ovary were obtained, every fifth section was stained with $\mathrm{H} \& \mathrm{E}$, and those sections were inspected. Sections that went through the center of the follicle (largest cross section) were categorized by using a modified follicle classification developed by Pedersen and Peters (14). This classification describes three distinct types of follicles, whose development is gonadotropin dependent (Figure 1c): class $5 b$ follicles with more than three layers of granulosa cells, with or without a very few fluid pockets and the oocyte visible; class 6 follicles, which have multiple fluid pockets with the oocyte visible; preovulatory follicles (class 7), which have a single continuous cavity containing follicular fluid surrounded by multiple layers of granulosa cells with the oocyte sometimes located in a well-formed cumulus stalk (class 8). A maximum of three follicles, each belonging to one of the three classes, per contralateral ovary could be selected for further characterization by immunohistochemical or immunofluorescent means. Such follicles of interest also had to meet a second condition to become eligible for evaluation: they had to be nonatretic. Atretic follicles can be differentiated from nonatretic follicles by the number of apoptotic granulosa cells. Atretic follicles are characterized by a high number of apoptotic granulosa cells (>10\%), and their fate is mostly elimination by atresia (15). Nonatretic follicles ( $<10 \%$ apoptotic cells) have the potential to develop into preovulatory follicles. H\&E staining allows identification of such nonatretic or "healthy" follicles. These follicles contain a normalshaped oocyte surrounded by granulosa cells that were regularly apposed on an intact basement membrane with a normal appearance of granulosa cell nuclei without signs of pyknosis (5). To confirm the correct histological assessment an adjacent section of the same follicle was stained for apoptosis by immunohistochemical means (see below). An H\&E-stained cross section through a follicle meeting all the inclusion criteria is called the index section and is given the number 0 . 
Sections immediately preceding and following the index section are identified by a number preceded by a minus $(-)$ or plus $(+)$ sign.

Immunohistochemistry. Vascular endowment of a follicle of interest was evaluated in the tissue sections immediately following the index section by staining against PECAM (16) using immunohistochemistry (section +1$)$ or immunofluorescence (section +2$)$, according to the manufacturer's instructions (PharMingen, San Diego, California, USA). To detect signals immunohistochemically, peroxidase staining was performed with diaminobenzidine tetrahydrochloride (DAB) as a chromogen (brown color) (DAB substrate kit; Vector Laboratories, Burlington, California, USA). Counterstaining to detect nuclei was performed with hematoxylin. Detection by immunofluorescent means was performed by using a fluorescent conjugate of streptavidin (Alexa Fluor 488; Molecular Probes Inc., Eugene, Oregon, USA), which emits green light. Nuclear staining was performed with DAPI (Vector Laboratories).

Granulosa cell proliferation $($ section +3 ) was identified with a biotinylated mouse anti-BrdU primary $\mathrm{Ab}$ (BrdU Zymed kit; Zymed Laboratories Inc., South San Francisco, California, USA) following the manufacturer's instruction. After incubating with streptavidin-peroxidase for 10 minutes at $25^{\circ} \mathrm{C}, \mathrm{DAB}$ (brown color) was used as a chromogen for single labeling (Vector Laboratories). Section +4 was stained with hematoxylin, which allowed counting the total number of granulosa cells using a computer program (see below). For dual labeling to identify proliferating endothelial cells, slides were first incubated with anti-PECAM Ab as described above and visualized with either DAB (brown color) for immunohistochemistry (section -1 ) or with Alexa Fluo 488 (green color) for immunofluorescence (Molecular Probes Inc.) (section -2). This was followed by incubation of the same sections with $\mathrm{Ab}$ against $\mathrm{BrdU}$ as described above and developed with either the chromogen VIP (purple color) for immunohistochemistry (Vector Laboratories (section -1) or with Alexa Fluor 594 (red color) (Molecular Probes Inc.) for immunofluorescence (section-2). Proliferating endothelial cells were identified as purple areas surrounded by a brown area in immunohistochemistry or as yellow areas through superposition of green and red color. For quality assurance, a Wilms tumor (provided by Jessica Kandel, Columbia University, New York, New York, USA) was included, because proliferating endothelial cells are frequent in tumors (data not shown).

Apoptotic cells (section -3) were detected by TUNEL, using an in situ detection kit (ApopTag; Intergen Co., Purchase, New York, New York, USA), according to the manufacturer's instruction. Testing for apoptosis was used to verify that individual follicles selected for immunohistochemical evaluation were nonatretic. In addition, the ratio of atretic versus nonatretic follicles was evaluated in a randomly selected section from the contralateral ovaries for the different experimental con- ditions. For quality assurance a Wilms tumor treated with antiangiogenic agent (provided by Jessica Kandel) was included, because apoptotic cells are frequent in such treated tumors (data not shown).

Type and number of follicles, whose growth is gonadotropin dependent. Gonadotropin-dependent follicle growth starts at the advanced preantral or early antral stage and culminates in the development of mature antral follicles ready to ovulate (9). The modified classification of follicle development by Pedersen and Peters (14) (Figure 1c) was used to count the total number of class 5 b, class 6 , and preovulatory follicles contained in one whole ovary from each animal. Such a numerical approach allowed us to compare the dynamics of gonadotropin-dependent follicle development between the different experimental groups over time. To avoid double counting, only follicles in which the nucleus of the oocyte was clearly visible were counted. Quantification was performed by two investigators in a blinded fashion, and the coefficient of correlation for follicle counts between the two investigators was 0.88 .

Quantification by immunohistochemistry. Quantitative analysis of PECAM, BrdU (proliferation), and $3^{\prime}$-end TUNEL labeling (apoptosis) was performed using an image analysis system linked to a Nikon Eclipse E800 camera, and the data were processed using ImagePro Plus Version 4.01 (Media Cybernetics, Silver Spring, Maryland, USA). Follicles of interest were identified, and the theca and granulosa cell compartments were outlined and analyzed separately.

Vasculature identified by PECAM labeling. Quantification of vasculature (section +1 ) present in individual follicles belonging to class $5 \mathrm{~b}$, class 6 , or the preovulatory stage from the contralateral ovary of each animal was performed whenever possible. The total area of the theca cell compartment was outlined per largest cross section of each follicle of interest and was measured at $\times 300$ (class $5 \mathrm{~b}$ and class 6 ) or at $\times 200$ (preovulatory follicle) magnification, and the area was expressed as micrometers squared. Subsequently, the total endothelial cell area, that is, PECAM-positive cells located within this compartment, were identified (total amount of vasculature), and the area was expressed as micrometers squared. Vascular density expressed as percentage was calculated by forming a ratio of PCEAM-positive area divided by the total theca layer area multiplied by 100 .

Proliferating cells identified by BrdU labeling. Cross sections through follicles of class $5 b$ or 6 were analyzed at a magnification of $\times 300$ and preovulatory follicles at $\times 200$.

Granulosa cells. Granulosa cell proliferation (section +3 ) was quantified in the same follicles whose vasculature had been analyzed. The image analysis system was set up to measure the number of dark-stained nuclei (BrdU positive) using the segmentation part of the computer program. The adjacent section, which was stained with hematoxylin, allowed for counting of the total number of granulosa cells identified as blue-stained nuclei in the follicle of interest. A granulosa cell proliferation index 
Table 1

Effect of PMSG or PMSG plus anti-VEGFR-2 Ab on follicular number per ovary diameter

\begin{tabular}{|c|c|c|c|}
\hline Experimental groups & Class 5b & Class 6 & Class $7 / 8$ \\
\hline Baseline & $9 \pm 1.1$ & none & none \\
\hline \multicolumn{4}{|c|}{24 hours after PMSG injection } \\
\hline $\begin{array}{l}\text { Control } \\
\text { Stimulation } \\
\text { Treatment }\end{array}$ & $\begin{array}{r}10.3 \pm 1.2 \\
11.5 \pm 1.4 \\
8.9 \pm 1.5\end{array}$ & $\begin{array}{c}\text { none }^{\mathrm{A}} \\
8.5 \pm 1.1^{\mathrm{B}} \\
3.3 \pm 0.9^{\mathrm{D}}\end{array}$ & $\begin{array}{l}\text { none } \\
\text { none } \\
\text { none }\end{array}$ \\
\hline \multicolumn{4}{|c|}{48 hours after PMSG injection } \\
\hline $\begin{array}{l}\text { Control } \\
\text { Stimulation } \\
\text { Treatment }\end{array}$ & $\begin{array}{r}9.5 \pm 1.3 \\
10.8 \pm 1.5 \\
11.1 \pm 1.4\end{array}$ & $\begin{array}{c}\text { none }^{\mathrm{A}} \\
11.5 \pm 1.6 \\
10.2 \pm 1.6^{\mathrm{D}}\end{array}$ & $\begin{array}{c}\text { none }^{\mathrm{A}} \\
4.1 \pm 0.3^{\mathrm{C}} \\
\text { none }\end{array}$ \\
\hline \multicolumn{4}{|c|}{72 hours after PMSG injection } \\
\hline $\begin{array}{l}\text { Control } \\
\text { Stimulation } \\
\text { Treatment }\end{array}$ & $\begin{array}{l}10.8 \pm 1.2 \\
11.5 \pm 1.9 \\
10.1 \pm 1.5\end{array}$ & $\begin{array}{c}\text { none }^{\mathrm{A}} \\
12.5 \pm 2.1 \\
8.9 \pm 1.1^{\mathrm{D}}\end{array}$ & $\begin{array}{c}\text { none }^{\mathrm{A}} \\
9.5 \pm 2.1^{\mathrm{C}} \\
0.8 \pm 0.1\end{array}$ \\
\hline
\end{tabular}

Values are mean \pm SE. Control versus stimulation: ${ }^{A} P<0.01$; stimulation versus treatment: ${ }^{\mathrm{B} P}<0.05,{ }^{C} P<0.01$; control versus treatment: $\mathrm{D} P<0.01$.

was formed (i.e., BrdU-positive cells expressed as a percentage of the total number of granulosa cells from the cross section of the follicle of interest). Because the number of granulosa cells per largest cross section correlates closely with the total number of granulosa cells for the same follicle, this number is representative of the total number of granulosa cells per follicle (17).

Proliferating endothelial cells. Proliferating endothelial cells (section -1 ) were identified manually by colocalization of BrdU and PECAM, because the automated image analysis program failed to identify double-labeled cells reliably. Because the number of dividing endothelial cells in developing follicles was generally low, a qualitative approach was used, and the follicles of interest were classified into two categories based on the presence $(+)$ or absence (-) of dividing endothelial cells.

Atresia. Atresia, a default pathway to eliminate follicles, is most prominent in class $5 \mathrm{~b}$ follicles (9). To quantify the extent of atresia, an index was formed by counting the number of atretic follicles belonging to class $5 \mathrm{~b}$ and beyond divided by the total number of advanced secondary or tertiary (antral) follicles in ran- domly selected cross sections from each contralateral ovary. One cross section per ovary was analyzed, and therefore a total of ten cross sections from ten ovaries in the baseline, control, stimulation, and treatment group were evaluated.

Quantification of fluid-filled areas or antrum. The total area of the granulosa cell compartment plus fluid-filled spaces or antrum was outlined per largest cross section from each follicle (section 0 ) selected for evaluation. Fluid-filled areas were measured at $\times 300$ (class $5 \mathrm{~b}$ and class 6 ) or at $\times 200$ (preovulatory follicle) magnification, and the areas were expressed as micrometers squared.

Serum estradiol and progesterone. Blood samples were obtained by cardiopuncture and allowed to clot for 1 hour. The blood samples were then centrifuged $(1,000 \mathrm{~g})$ for 15 minutes at room temperature, and the serum was stored at $-20^{\circ} \mathrm{C}$. Estradiol and progesterone were measured with a ligand-labeled, competitive, chemiluminescent immunoassay (Diagnostic Products Corp., Los Angeles, California, USA). The interassay coefficients of variation were $11.9 \%$ and $11.1 \%$ for estradiol and progesterone, respectively.

Statistical analysis. Data are expressed as mean plus or minus the standard error. ANOVA (Kruskal-Wallis) and post hoc Dunn's multiple comparison were used for statistical analysis, with $P$ values less than 0.05 considered significant (11). For nonparametric testing, the $\chi$-square test was used.

\section{Results}

PMSG administration to HX mice stimulates follicular development and angiogenesis. We counted the total number of class 5 b, class 6 , and preovulatory follicles present in whole ovaries from animals belonging to baseline (untreated), control (saline), or stimulation (PMSG plus saline) group to characterize the dynamics of follicle development over time. Follicles of the class $5 \mathrm{~b}$ type dominated in ovaries from the baseline and the control group (experimental days 1, 2, and 3), whereas follicles of advanced development (those with a significant amount of antrum) were rarely seen (Figure 1a, top panel; Table 1). Little variation in the number of such

Table 2

Effect of PMSG or PMSG plus anti-VEGFR-2 Ab on follicular vasculature $\left(\mu \mathrm{m}^{2}\right)$ and blood vessel density $\left(\mu \mathrm{m}^{2} /\right.$ theca layer area $)$

\begin{tabular}{|c|c|c|c|c|c|c|}
\hline \multirow[b]{2}{*}{ Experimental groups } & \multicolumn{3}{|c|}{ Vasculature $\left(\mu \mathrm{m}^{2}\right)$} & \multicolumn{3}{|c|}{ Blood vessel density (\%) } \\
\hline & Class $5 b$ & Class 6 & Class $7 / 8$ & Class $5 \mathrm{~b} / 6$ & Class 6 & Class $7 / 8$ \\
\hline \multicolumn{7}{|c|}{24 hours after PMSG injection } \\
\hline $\begin{array}{l}\text { Control } \\
\text { Stimulation } \\
\text { Treatment }\end{array}$ & $\begin{array}{l}1,824 \pm 383^{B} \\
2,585 \pm 423^{D} \\
1,598 \pm 210\end{array}$ & $\begin{array}{c}\text { none } \\
2,944 \pm 234^{\mathrm{D}} \\
1,874 \pm 179\end{array}$ & $\begin{array}{l}\text { none } \\
\text { none } \\
\text { none }\end{array}$ & $\begin{array}{l}13.9 \pm 2.9^{A} \\
20.5 \pm 2.1^{C} \\
11.5 \pm 1.1\end{array}$ & $\begin{array}{c}\text { none } \\
23.4 \pm 1.2 \\
8.5 \pm 2.1\end{array}$ & $\begin{array}{l}\text { none } \\
\text { none } \\
\text { none }\end{array}$ \\
\hline \multicolumn{7}{|c|}{48 hours after PMSG injection } \\
\hline $\begin{array}{l}\text { Control } \\
\text { Stimulation } \\
\text { Treatment }\end{array}$ & $\begin{array}{l}1,681 \pm 245^{A} \\
2,199 \pm 344^{C} \\
1,778 \pm 485\end{array}$ & $\begin{array}{c}\text { none } \\
3,211 \pm 297^{D} \\
1,590 \pm 165\end{array}$ & $\begin{array}{c}\text { none } \\
4,355 \pm 875 \\
\text { none }\end{array}$ & $\begin{array}{l}12.5 \pm 3.1^{\mathrm{A}} \\
19.1 \pm 3.8^{\mathrm{C}} \\
13.1 \pm 2.1\end{array}$ & $\begin{array}{c}\text { none } \\
17.2 \pm 2.1^{\mathrm{D}} \\
9.5 \pm 1.7\end{array}$ & $\begin{array}{c}\text { none } \\
18.8 \pm 6.0 \\
\text { none }\end{array}$ \\
\hline \multicolumn{7}{|c|}{72 hours after PMSG injection } \\
\hline $\begin{array}{l}\text { Control } \\
\text { Stimulation } \\
\text { Treatment }\end{array}$ & $\begin{array}{l}1,581 \pm 291^{A} \\
2,345 \pm 301^{C} \\
1,721 \pm 281\end{array}$ & $\begin{array}{c}\text { none } \\
3,467 \pm 391^{\mathrm{D}} \\
1,677 \pm 245\end{array}$ & $\begin{array}{c}\text { none } \\
5,347 \pm 442 \\
\text { none }\end{array}$ & $\begin{array}{l}13.1 \pm 2.4^{\mathrm{A}} \\
21.1 \pm 4.7^{\mathrm{C}} \\
14.5 \pm 1.9\end{array}$ & $\begin{array}{c}\text { none } \\
18.8 \pm 3.4^{\mathrm{C}} \\
10.1 \pm 2.1\end{array}$ & $\begin{array}{c}\text { none } \\
16.9 \pm 3.8 \\
\text { none }\end{array}$ \\
\hline
\end{tabular}

Values are mean $\pm \mathrm{SE}$. Control versus stimulation: ${ }^{\mathrm{A} P}<0.05,{ }^{\mathrm{B}} P<0.01$; stimulation versus treatment: ${ }^{\mathrm{C}} P<0.05,{ }^{\mathrm{D}} P<0.01$. 
follicles per ovary was observed between the baseline group and the control groups (Table 1). Preovulatory follicles (large, continuous fluid-filled cavity) dominated in stimulation group ovaries after a 72-hour exposure to gonadotropins $(9.5 \pm 2.1$ follicles per ovary) (Figure $1 \mathrm{a}$, middle panel; Table 1 ), and few corpora lutea were seen as well ( $2.5 \pm 0.2$ follicles per ovary). Preovulatory follicles were absent 24 hours after PMSG administration and started to appear 48 hours after PMSG administration ( $4.1 \pm 0.3$ follicles per ovary). The total number of class $5 \mathrm{~b}$ follicles 24 hours, 48 hours, and 72 hours after PMSG administration in the stimulation group was similar to the number seen in the control group (Table 1). Class 6 follicles (larger areas of follicular fluid but no continuous fluid-filled cavity) were first seen 24 hours after PMSG administration ( $8.5 \pm 1.1$ follicles per ovary). The number of this type of follicle continued to increase 48 hours after PMSG administration (11.5 \pm 1.6 follicles per ovary), but no further rise was seen 72 hours after PMSG administration (12.5 \pm 2.1 follicles per ovary).

Subsequently, an analysis of the vasculature in individual follicles belonging to class $5 \mathrm{~b}$, class 6 , or the preovulatory stage was performed in the contralateral ovaries of all animals in baseline, control, or stimulation groups. There was no significant difference in the total amount of vasculature and blood vessel density between class $5 \mathrm{~b}$ follicles belonging either to the baseline or the different control groups (experimental day 1, 2, or 3) (Table 2). Endothelial cell proliferation was generally absent in such class $5 \mathrm{~b}$ follicles. In the stimulation group, the total amount of theca layer vasculature was highest in preovulatory follicles. Class 6 follicles had less vasculature than preovulatory follicles, but more than class $5 \mathrm{~b}$ follicles (Table 2). Therefore, with advancement of follicle development, the total amount of vasculature continued to increase (Table 1; Figure 2 and Figure 3). Blood vessel density was similar between different types of stimulation group follicles (class $5 \mathrm{~b}$ to preovulatory) for all the time points studied and ranged from $17 \%$ to $23 \%$. It is of note that the total amount of vasculature and blood vessel density in class $5 \mathrm{~b}$ stimulation group follicles was always higher when compared with follicles of the same type found in baseline or control group ovaries $(<15 \%)$ (Figure 2 and Table 2 ). This increase in vascular density of stimulation group $5 \mathrm{~b}$ follicles over control (Figure 2) indicates that one of the initial steps of gonadotropin-dependent follicular development is an increase in follicular vasculature, which in turn increases follicular blood supply and secures further follicular growth. In general, the increase in the amount of follicular vasculature and vascular density in stimulation group follicles is due to endothelial cell proliferation because dividing endothelial cells are consistently observed in the theca layer of follicles whose growth is gonadotropin dependent (Figure 2 and $3 ; P<0.01$ ).

Our data demonstrate that the immature, HX mouse model is well suited to evaluate the importance of intraovarian follicular angiogenesis for gonadotropin- dependent follicular development. Ovaries from animals HX before 24 days of age contain mostly immature follicles, with the most advanced cohort belonging to class 5b. Maturation of follicles beyond this stage accompanied by active follicular angiogenesis is induced by the administration of exogenous gonadotropins (11). Hypophysectomy excludes the possibility that alterations in hypothalamus/pituitary gland hormone secretion due to changes in feedback or due to a direct effect of anti-VEGFR-2 Ab on this unit will affect ovarian function.

VEGFR-2 activity is required for gonadotropin-induced follicular development and angiogenesis. We next examined the effect of VEGFR-2-neutralizing $\mathrm{Ab}$, an antiangiogenic agent (13), on the dynamics of gonadotropindependent follicle development and follicular angiogenesis in animals stimulated with PMSG. Class 6 follicles (multiple fluid pockets) dominated in treatment group ovaries 48 hours $(10.2 \pm 1.6$ follicles per ovary) and 72 hours (8.9 \pm 1.1 follicles per ovary; Figure 1a, lower panel) after PMSG. Some class 6 follicles were already seen 24 hours after PMSG stimulation (3.3 \pm 0.9 follicles per ovary) (Figure 1). Preovulatory follicles were mostly absent at all time points examined in treatment group ovaries (Figure 1; Table 1). Because the number of class 6 follicles is approximately the same 48 hours and 72 hours after PMSG administration, and preovulatory follicles were rarely

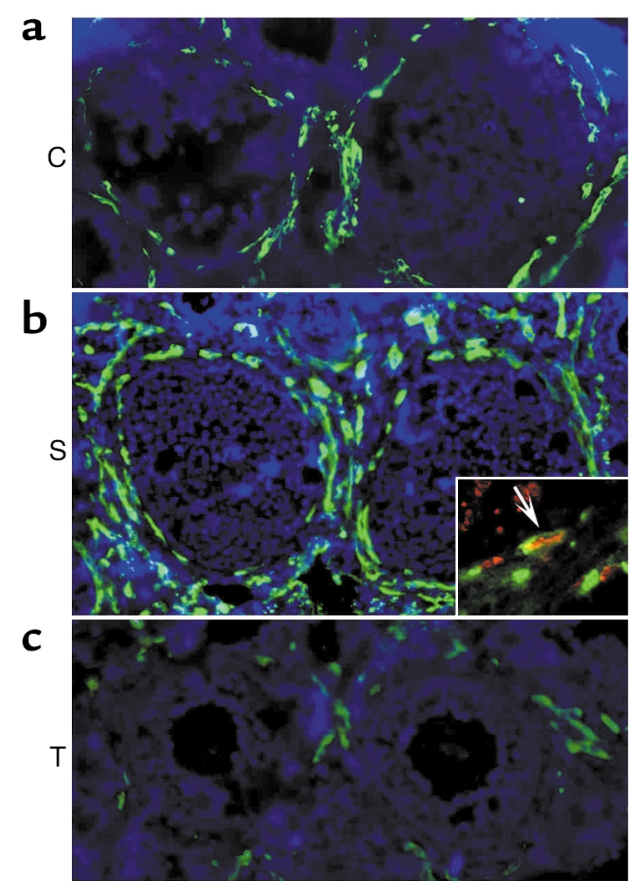

Figure 2

(a-c) Immunofluorescent staining of follicular vasculature of class $5 \mathrm{~b}$ follicles on experimental day 1. (a) C, control group follicle; (b) $\mathrm{S}$, PMSG-stimulated follicle; (c) T, treatment group follicle. Insert in b shows proliferating endothelial cells (arrow). Green: PECAM staining for blood vessels; red: BrdU staining for cell proliferation; yellow: combined staining indication of blood vessel proliferation. 


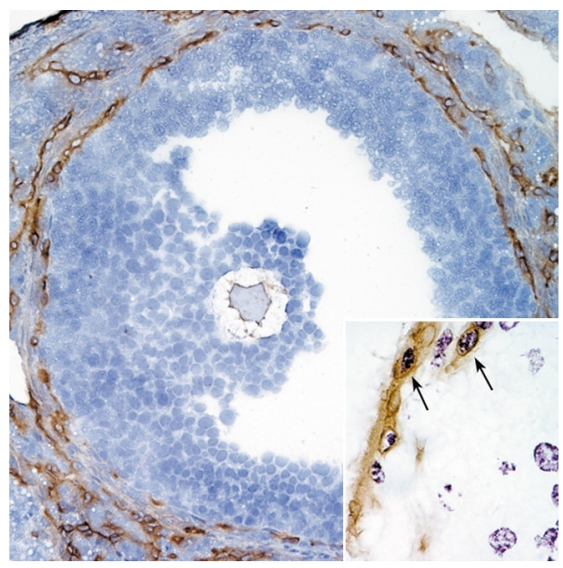

Figure 3

Immunohistochemical staining of follicular vasculature of a preovulatory follicle from the stimulation group ovary on experimental day 3 . Counterstaining with hematoxylin. Insert shows proliferating endothelial cells (arrows). Brown: PECAM staining for blood vessels; purple: $\mathrm{BrdU}$ staining for cell proliferation; brown surrounding purple: combined staining indication of blood vessel proliferation.

seen, one can conclude that administration of anti-VEGFR-2 Ab caused an arrest of gonadotropindependent follicle growth at the class 6 stage. The total number of class $5 \mathrm{~b}$ follicles per ovary counted on experimental day 1, 2, and 3 after combined PMSG and anti-VEGFR-2 Ab administration in the treatment group were similar to the numbers seen in the stimulation, control, and baseline group (Table 1).

Subsequently, an analysis of the vasculature in individual follicles belonging to class $5 \mathrm{~b}$ or class 6 was performed in the contralateral ovaries of all treatment group animals. The total amount of vasculature of class 6 follicles was similar to the amount seen in class $5 \mathrm{~b}$ follicles for all time points examined (Table 1). Thus, advancement of follicle development to class 6 from class $5 \mathrm{~b}$ occurred without an increase in the total amount of theca layer vasculature. It is of note that vascular density in class 6 decreased slightly when compared with the density of class $5 \mathrm{~b}$ follicles (Table 2 ). This observation may be explained by the fact that the total area of the theca layer tissue increased due to theca cell proliferation stimulated by PMSG in treatment group follicles (unpublished observation). In contrast the amount of theca layer vasculature did not change, because anti-VEGFR-2 Ab inhibited endothelial cell proliferation. Therefore, the ratio of theca cells to endothelial cells changed, which in turn caused this decrease in vascular density.

Blocking VEGFR-2 activity interferes with antrum formation. Mature, preovulatory follicles are characterized by the development of a large, single, central fluid-filled antrum. To assess how anti-VEGFR-2 Ab treatment affects gonadotropin-dependent cavity formation activity, we compared the area of cavity of the most advanced follicles between control, stimulation, and treatment groups after a 72-hour exposure to a sham injection or PMSG. The area of cavity was highest in the most-developed follicles of the stimulation group (Figure $1 \mathrm{~b}$, middle ovary) $\left(49,920 \pm 5,678 \mu \mathrm{m}^{2}\right)$, whereas it was very low in the most-developed follicles from the control group (upper ovary) $\left(2,074 \pm 924 \mu \mathrm{m}^{2}\right.$; $P<0.01)$. This difference is explained by the fact that multiple preovulatory follicles with large, continuous fluid-filled cavities were seen in PMSG-treated animals, whereas no such follicles were detected in control group ovaries. Some cavity formation activity occurred in the most developed follicles from the treatment group as demonstrated by many fluid-filled pockets among granulosa cells in class 6 follicles (Figure 1). The area of fluid-filled pockets measured 11,707 $\pm 1,144$ $\mu \mathrm{m}^{2}$, which is significantly lower when compared with the most-developed follicles in the stimulation group $(P<0.01)$, but higher when compared with class $5 \mathrm{~b}$ control group follicles $(P<0.01)$. This observation indicates some cavity formation in treatment group ovaries, but an inability to form large, single, confluent cavities as seen in preovulatory follicles from stimulation group ovaries.

VEGFR-2 activity is required for gonadotropin-dependent increase in granulosa cell proliferation. Significant granulosa cell proliferation was detected in all nonatretic class $5 \mathrm{~b}$ follicles in baseline and control group ovaries. The proliferation index of individual class $5 \mathrm{~b}$ control group follicles selected for immunohistochemical evaluation was between $21 \%$ and $27 \%$ (dividing cells per total cell number multiplied by 100) (Figure 4; Table 3) and did not change over time (experimental day 1, 2, and 3 ). In comparison with control follicles, the proliferation index more than doubled in class $5 \mathrm{~b}$ and class 6 follicles in the stimulation group when analyzed 24 hours, 48 hours, and 72 hours after initiation of PMSG stimulation fluctuating between $40 \%$ and 50\% (Figure 3; Table 3). It declined somewhat in preovulatory follicles (Table 3). In contrast, in the treatment group proliferative activity of granulosa cells from class 5 b or class 6 follicles was around $30 \%$ and did not change significantly over time. Therefore, proliferative activity in treatment group fol-

\section{Table 3}

Effect of PMSG or PMSG plus anti-VEGFR-2 Ab on granulosa cell proliferation

\begin{tabular}{|c|c|c|c|}
\hline Experimental groups & Class $5 \mathrm{~b} / 6$ & Class 6 & Class $7 / 8$ \\
\hline \multicolumn{4}{|c|}{24 hours after PMSG injection } \\
\hline $\begin{array}{l}\text { Control } \\
\text { Stimulation } \\
\text { Treatment }\end{array}$ & $\begin{array}{l}0.23 \pm 0.03^{A} \\
0.45 \pm 0.05 \\
0.37 \pm 0.03^{C}\end{array}$ & $\begin{array}{c}\text { none } \\
0.51 \pm 0.06^{\mathrm{B}} \\
0.31 \pm 0.04\end{array}$ & $\begin{array}{l}\text { none } \\
\text { none } \\
\text { none }\end{array}$ \\
\hline \multicolumn{4}{|c|}{48 hours after PMSG injection } \\
\hline $\begin{array}{l}\text { Control } \\
\text { Stimulation } \\
\text { Treatment }\end{array}$ & $\begin{array}{l}0.27 \pm 0.03^{\mathrm{A}} \\
0.49 \pm 0.04^{\mathrm{B}} \\
0.32 \pm 0.04\end{array}$ & $\begin{array}{c}\text { none } \\
0.52 \pm 0.06^{\mathrm{B}} \\
0.29 \pm 0.03\end{array}$ & $\begin{array}{c}\text { none } \\
0.39 \pm 0.05 \\
\text { none }\end{array}$ \\
\hline \multicolumn{4}{|c|}{72 hours after PMSG injection } \\
\hline $\begin{array}{l}\text { Control } \\
\text { Stimulation } \\
\text { Treatment }\end{array}$ & $\begin{array}{l}0.21 \pm 0.03^{A} \\
0.53 \pm 0.05^{B} \\
0.30 \pm 0.02^{C}\end{array}$ & $\begin{array}{c}\text { none } \\
0.54 \pm 0.06^{\mathrm{B}} \\
0.33 \pm 0.04\end{array}$ & $\begin{array}{c}\text { none } \\
0.41 \pm 0.04 \\
\text { none }\end{array}$ \\
\hline
\end{tabular}

Values are mean \pm SE. Control versus stimulation: ${ }^{A} P<0.01$; stimulation versus treatment: ${ }^{\mathrm{B}} P<0.05$, ; control versus treatment: ${ }^{C} P<0.05$. 
a
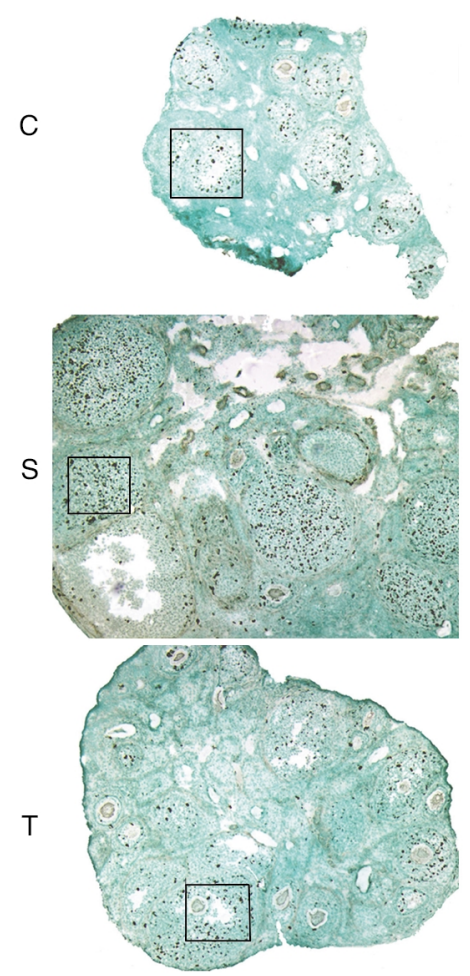

b
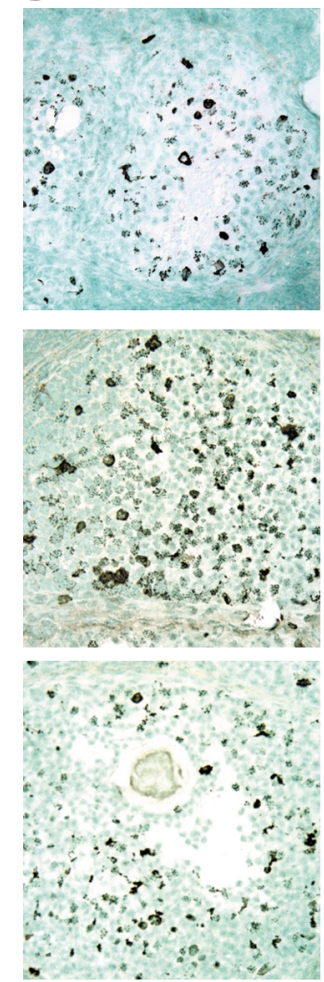

\section{Figure 4}

Granulosa cell proliferation on experimental day 1. (a) Low-power magnification of an ovarian cross section; (b) intermediate-power magnification of class 5 b or early class 6 follicles from each experimental group. C, control group follicle; S, PMSG-stimulated follicle; $\mathrm{T}$, treatment group follicle. Proliferation is seen as black dots. Counterstaining is with methyl-green.

licles was higher when compared with control group follicles, but less when compared with stimulation group follicles. The difference in proliferative indices measured for the same follicle stage at each experimental day between the three groups was statistically significant most of the time $(P<0.05$; Table 3$)$.

Blocking VEGFR-2 activity interferes with gonadotropinmediated prevention of atresia. Slightly more than half of all class $5 \mathrm{~b}$ follicles were atretic in baseline (data not shown) and control group ovaries (experimental day 3), as demonstrated by the presence of many follicles in which more than $10 \%$ of granulosa cells are apoptotic (Figure 5 ; Table 4). In contrast, the ovaries of stimulation group animals evaluated 72 hours after the PMSG injection rarely displayed atretic follicles (Figure 5; Table 4). Thus, treatment with gonadotropins is able to reverse the fate of preexisting atretic follicles. Gonadotropins in the presence of anti-VEGFR-2 Ab (treatment group) reduced the number of atretic follicles to about 34\% (number of atretic follicles per total number of follicles belonging to class $5 \mathrm{~b}$ or higher multiplied by 100) when compared with baseline and control ovaries. The differences between the three experimental groups were statistically significant $(P<0.05$; Table 4$)$

Treatment with anti-VEGFR-2 $A b$ interferes with gonadotropin-induced estradiol/progesterone secretion and ovarian/uterine growth. As seen in Table 4, gonadotropins induced a threefold increase in serum estradi$\mathrm{ol}$ and progesterone concentrations in stimulation group animals compared with control $(P<0.05)$ when measured 72 hours after the PMSG injection. A slight but nonsignificant rise in estradiol and progesterone secretion was observed in the anti-VEGFR-2 treatment group when compared with control. Induction of preovulatory follicular development with PMSG was associated with a $50 \%$ increase in ovarian weight when compared with animals not hormonally treated (control group: $6.0 \pm 0.6 \mathrm{mg}$; PMSG-treated group: $10.1 \pm 1.1 \mathrm{mg} ; P<0.05)$. Treatment with anti-mouse VEFGR-2 Ab in the presence of PMSG (anti-VEGFR-2 group) resulted in a complete suppression of the ovarian weight gain seen for the PMSG-stimulated group (treatment: $6.2 \pm 0.5 \mathrm{mg} ; P<0.05$ ). PMSG administration also induced a nearly threefold increase in the uterine weight in the stimulation group compared with control evaluated 72 hours after PMSG or sham injection (control: $15.7 \pm 4.2 \mathrm{mg}$; PMSG-stimulated group: $50.3 \pm 7.5 \mathrm{mg} ; P<0.01$ ). In the anti-VEGFR-2 treatment group, uterine weight failed to increase to levels seen in PMSG-stimulated animals $(28.9 \pm 3.5 \mathrm{mg} ; P<0.05)$.

\section{Discussion}

Administration of an anti-VEGFR-2 Ab (DC101) inhibits gonadotropin-dependent follicular angiogenesis, which in turn blocks development of mature antral follicles. We conclude that the intraovarian VEGF/VEGFR-2 pathway plays a critical mediatory role in the process of gonadotropin-mediated follicular development.

Gonadotropins drive follicular development, and a component of this function is derived from the effects of gonadotropins on the vasculature of the ovary. Administration of exogenous gonadotropins in the form of PMSG triples the amount of follicular vasculature during follicular development from immature class $5 \mathrm{~b}$ to the mature preovulatory stage. Increase in follicular vasculature is due to active follicular angiogenesis, which is frequently detected in growing follicles in the form of dividing endothelial cells. Administration of a blocking $\mathrm{Ab}$ against VEGFR-2 partially inhibits

\section{Table 4}

Effect of PMSG or PMSG plus anti-VEGFR-2 Ab on atresia, steroids, and ovarian and uterine weight 72 hours after PMSG injection

\begin{tabular}{lccc}
\hline & Control & Stimulation & Treatment \\
& $0.54 \pm 0.04^{\mathrm{B}}$ & $0^{\mathrm{D}}$ & $0.34 \pm 0.07^{\mathrm{E}}$ \\
Atresia index & $34 \pm 3.5^{\mathrm{B}}$ & $90.4 \pm 11.1^{\mathrm{D}}$ & $48 \pm 5.6$ \\
Estradiol $(\mathrm{pg} / \mathrm{ml})$ & $0.3 \pm 0.09^{\mathrm{A}}$ & $1.1 \pm 0.37^{\mathrm{C}}$ & $0.3 \pm 0.08$ \\
Progesterone $(\mathrm{ng} / \mathrm{ml})$ & $6 \pm 0.65^{\mathrm{A}}$ & $10.1 \pm 1.1^{\mathrm{C}}$ & $6.2 \pm 0.54$ \\
Ovarian weight $(\mathrm{mg})$ & $15.7 \pm 4.2^{\mathrm{B}}$ & $50.3 \pm 7.4^{\mathrm{C}}$ & $28.9 \pm 3.5$ \\
Uterine weight $(\mathrm{mg})$ & $15.9 \pm$ \\
\hline
\end{tabular}

Values are mean \pm SE. Control versus stimulation: ${ }^{A} P<0.05,{ }^{B} P<0.01$; stimulation versus treatment: ${ }^{C} P<0.05,{ }^{D} P<0.01$; control versus treatment: ${ }^{\mathrm{E}} P<0.05$. 

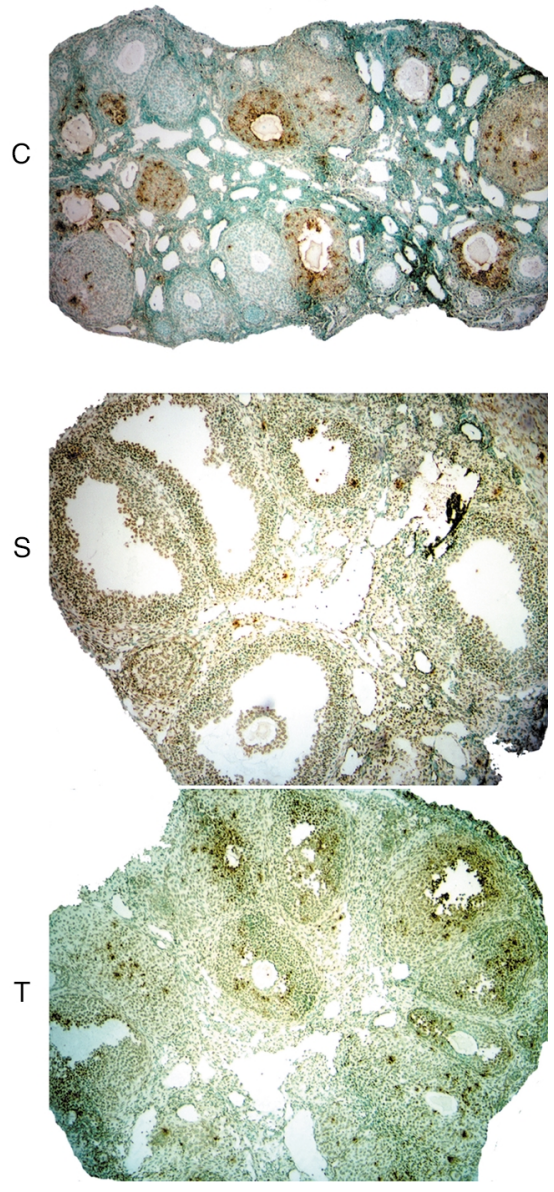

\section{Figure 5}

(a-c) Follicular atresia 72 hours after sham or PMSG injection. (a) C, control group follicle; (b) S, PMSG-stimulated follicle; (c) T, treatment group follicle. Atretic follicles are characterized by multiple, apoptotic granulosa cells, which appear as brown dots. Counterstaining with methyl-green.

gonadotropin-dependent follicular development; that is, class $5 \mathrm{~b}$ follicles can advance to class 6 but not to the preovulatory stage. It is of note that the total amount of vasculature seen in class 6 follicles is similar to that seen in class $5 \mathrm{~b}$ in the treatment group. This observation suggests that the $A b$, while not affecting survival of preexisting vasculature, prevented significant endothelial cell proliferation. Indeed, dividing endothelial cells were rarely seen in such follicles. Thus, in the treatment group gonadotropin-driven follicular development can partially progress without concomitant increase in follicular vasculature. This partial progression may be a result of sufficient delivery of PMSG and other substances by preexisting vasculature to allow such partial follicular development. On the other hand a substantial increase in follicular vasculature may be required to secure delivery of PMSG and other substances at levels sufficient to permit progression to the preovulatory stage. Based on our findings, we hypothesize that the failure to increase follicular blood supply by increasing the follicular amount of vasculature by angiogenesis prohibits an increase in the delivery of substances essential for follicle development to the preovulatory stage.

Due to the specificity of the $\mathrm{Ab}$ (i.e., it exclusively blocks function of VEGFR-2), one can define the intraovarian role of the VEGF/VEGFR-2 pathway for gonadotropin-dependent folliculogenesis. Based on our findings, the gonadotropin-dependent induction of angiogenesis in follicles very likely occurs by indirect means. VEGF is produced by granulosa and theca cells $(1,2,18,19)$, and it has been reported that VEGF expression is stimulated by gonadotropins (20). VEGFR-2 is consistently expressed on endothelial cells found in the theca layer of follicles (19) (unpublished observation). Thus, gonadotropins likely increase VEGF production and secretion in follicular epithelial cells, which in a paracrine manner acts on VEGFR-2 located on theca layer endothelial cells to induce angiogenesis. While the VEGF/VEGFR-2 pathway seems to be critical for endothelial cell proliferation, it seems to be of lesser importance to secure survival of preexisting follicular vasculature. This finding is congruent with fact that follicular blood vessel are mature vessels, i.e., endothelial cells are surrounded by pericytes (unpublished observation), which are less dependent on the VEGF/VEGFR-2 pathway for survival (8). Results from our study help in the interpretation of results obtained from functional studies inactivating VEGF by using "VEGF-trap" $(2,5)$ or specific Ab's against VEGF (6) as antiangiogenic agents. It is likely that the treatment effects are due to the inability of VEGF to activate VEGFR-2. As VEGFR-2 mediates VEGF-dependent angiogenesis, it is less likely that VEGFR-1 plays an important role during this process. The role of other angiogenic factors, including angiopoietins acting through the Tie 2 receptor for gonadotropin-dependent follicle development, must be further defined $(2,4,5)$.

A mature, preovulatory follicle is characterized by the formation of a single, centrally located, continuous, fluid-filled antrum. The antrum develops in concert with the increase in vasculature surrounding the follicle. In HX mice PMSG completes the formation of such cavities 48 hours to 72 hours after initiation of stimulation. The formation of follicular cavities was completely absent in the control group, emphasizing its dependence on gonadotropins. Cavity formation activity mediated by gonadotropins is blocked by anti-VEGFR-2 Ab treatment, and only small, discontinuous fluid-filled pockets among granulosa cells are detected. What mechanisms are used to develop the fluid-filled antrum? VEGF is known to promote both endothelial cell proliferation and to regulate vascular permeability $(18,21)$; thus, one may propose that an increase in vascular permeability might contribute to the physiologic process of antrum formation (1). Our data support a role for VEGF acting through VEGFR-2 as one factor involved in this process $(1,22,23)$; however, it is difficult to experimentally segregate the mitogenic from permeability 
functions of VEGF. It is also possible that other gonadotropin-dependent regulators of vascular permeability such as angiopoietins $(1,23,24)$ and regulators of cell permeability such as aquaporins (25) also participate in the process of cavity formation.

An increase in cell mass through epithelial proliferation contributes to follicular growth during follicle maturation. In the stimulation group, PMSG reaching the growing follicle through preexisting and newly formed vessels stimulates proliferation of granulosa and theca cells during gonadotropin-dependent maturation beyond the level seen in the baseline and control group follicles. In the treatment group, follicular epithelial cells proliferate in response to gonadotropins, but at a much lower rate when compared with the stimulation group. Inhibition of blood vessel proliferation by angiogenesis blocking $\mathrm{Ab}$ limits the amount of PMSG reaching epithelial cells in the follicle, which explains why proliferation does not reach the level seen in the stimulation group. Therefore, inhibition of follicular angiogenesis not only inhibits gonadotropin-mediated antrum formation but also the level of proliferation of follicular epithelial cells.

In the absence of gonadotropins, the most advanced immature follicles belonging to class $5 \mathrm{~b}$ are gradually eliminated through atresia by cellular apoptosis (26). These follicles are continuously replaced with newly maturing follicles originating from a resting pool of follicles (9). In ovaries of HX mice more than half of class $5 \mathrm{~b}$ follicles were atretic. In contrast, atretic follicles were mostly absent in ovaries from HX animals stimulated with PMSG. It thus appears that follicular atresia in HX mice can be reversed or prevented by gonadotropin administration (15). An angiogenic block induced in the treatment group by anti-VEGFR-2 Ab partially prevented the gonadotropin-dependent reversal of atresia. We speculate that the failure to increase the blood supply through angiogenesis is a contributing factor preventing PMSG-dependent reversal of atresia in these ovaries. Thus, our data support that of other groups in hypothesizing that insufficient vascular support is one of the mechanisms that contributes to follicular atresia $(5,27)$.

Hormonal measures and weight parameters support the finding that anti-VEGFR-2 $\mathrm{Ab}$ inhibits gonadotropin-dependent ovarian follicular development. Estradiol and progesterone levels are significantly increased in the PMSG-stimulation group compared with control, but no such rise was observed in the anti-VEGFR-2treated group. This is secondary to a failure to increase follicular granulosa and theca cell numbers to levels seen in preovulatory follicles of stimulation group ovaries. Also, the amount of gonadotropins reaching treatment group follicles is less when compared with stimulation group follicles due to the differences in follicular vasculature. Ovarian weight did not increase significantly in anti-VEGFR-2-treated ovaries compared with gonadotropin-stimulated ovaries, most likely related to the absence of advanced stage follicles in treatment ovaries.
Uterine weight was significantly lower in treatment group animals compared with stimulation group uteri. This failure to increase uterine weight to levels seen in the stimulation group is likely secondary to decreased ovarian steroid production and secretion. An additional direct effect of angiogenic inhibitors on uterine growth, however, cannot be excluded.

In this study we haven chosen a new model, the prepuberally HX mouse, to delineate the importance of the VEGF/VEGFR-2 pathway for gonadotropindependent follicular development. In contrast to previous studies $(2,5-7)$ where feedback is active, our a model avoids interference by feedback when the antiangiogenic agents are given. Using such a design eliminates a confounding variable that could have an impact on the interpretation of results.

In summary, results obtained in our HX mouse model clearly demonstrate that high doses of exogenous gonadotropins are unable to induce optimal follicle development due to the intraovarian action of the anti-VEGFR-2 Ab. Follicular angiogenesis is critical for the development of gonadotropin-dependent, mature antral follicles from immature ones. Further research is needed to answer the question of whether the VEGF/VEGFR-2 pathway is also involved in the regulation of the formation of a follicular antrum through its action on vascular permeability. In addition, it will be important to understand what role other angiogenic regulators, such as angiopoietins, play in follicular angiogenesis and in follicle development.

\section{Acknowledgments}

The authors thank Ennian Xiao and Michel Ferin for helpful discussions and Alinda Barth for help in performing the assays. This work was supported by NIH grant K12 HD-01275 (to R. Zimmermann) and by NIH grant HL-62454 and American Heart Association grant AHA0150435N (to J. Kitajewski).

1. Geva, E., and Jaffe, R.B. 2000. Role of vascular endothelial growth factor in ovarian physiology and pathology. Fertil. Steril. 74:429-438.

2. Wulff, C., Wiegand, S.J., Saunders, P.T., Scobie, G.A., and Fraser, H.M. 2001. Angiogenesis during follicular development in the primate and its inhibition by treatment with truncated Flt-1-Fc (vascular endothelial growth factor Trap(A40)). Endocrinology. 142:3244-3254.

3. Geva, E., and Jaffe, R.B. 2000. Role of angiopoietins in reproductive tract angiogenesis. Obstet. Gynecol. Surv. 55:511-519.

4. Maisonpierre, P.C., et al. 1997. Angiopoietin-2, a natural antagonist for Tie2 that disrupts in vivo angiogenesis. Science. 277:55-60.

5. Wulff, C., Wilson, H., Wiegand, S.J., Rudge, J.S., and Fraser, H.M. 2002. Prevention of thecal angiogenesis, antral follicular growth, and ovulation in the primate by treatment with vascular endothelial growth factor Trap R1R2. Endocrinology. 143:2797-2807.

6. Zimmermann, R.C., et al. 2001. Short-term administration of antivascular endothelial growth factor antibody in the late follicular phase delays follicular development in the rhesus monkey. J. Clin. Endocrinol. Metab. 86:768-772.

7.Zimmermann, R.C., Xiao, E., Bohlen, P., and Ferin, M. 2002. Administration of anti-vascular endothelial growth factor receptor 2 antibody in the early follicular phase delays follicular selection and development in the rhesus monkey. Endocrinology. 143:2496-2502.

8. Ferrara, N., Gerber, H.-P., and LeCouter, J. 2003. The biology of VEGF and its receptors. Nat. Med. 9:669-676.

9. McGee, E.A., and Hsueh, A.J. 2000. Initial and cyclic recruitment of ovarian follicles. Endocr. Rev. 21:200-214.

10. Halpin, D.M., and Charlton, H.M. 1988. Effects of short-term injection of gonadotrophins on ovarian follicle development in hypogonadal 
(hpg) mice. J. Reprod. Fertil. 82:393-400.

11. Wang, X.N., and Greenwald, G.S. 1993. Hypophysectomy of the cyclic mouse. II. Effects of follicle-stimulating hormone (FSH) and luteinizing hormone on folliculogenesis, FSH and human chorionic gonadotropin receptors, and steroidogenesis. Biol. Reprod. 48:595-605.

12. Wang, X.N., and Greenwald, G.S. 1993. Hypophysectomy of the cyclic mouse. I. Effects on folliculogenesis, oocyte growth, and follicle-stimulating hormone and human chorionic gonadotropin receptors. Biol. Reprod. 48:585-594.

13. Zimmermann, R.C., Hartman, T., Bohlen, P., Sauer, M.V., and Kitajewski, J. 2001. Preovulatory treatment of mice with anti-VEGF receptor 2 antibody inhibits angiogenesis in corpora lutea. Microvasc. Res. 62:15-25.

14. Pedersen, T., and Peters, H. 1968. Proposal for a classification of oocytes and follicles in the mouse ovary. J. Reprod. Fertil. 17:555-557.

15. Hsueh, A.J., Billig, H., and Tsafriri, A. 1994. Ovarian follicle atresia: a hormonally controlled apoptotic process. Endocr. Rev. 15:707-724.

16. Vecchi, A., et al. 1994. Monoclonal antibodies specific for endothelial cells of mouse blood vessels. Their application in the identification of adult and embryonic endothelium. Eur. J. Cell. Biol. 63:247-254.

17. Pedersen, T. 1970. Follicle kinetics in the ovary of the cyclic mouse. Acta Endocrinol. (Copenh.). 64:304-323.

18. Gomez, R., Simon, C., Remohi, J., and Pellicer, A. 2002. Vascular endothelial growth factor receptor- 2 activation induces vascular permeability in hyperstimulated rats, and this effect is prevented by receptor blockade. Endocrinology. 143:4339-4348

19. Shweiki, D., Itin, A., Neufeld, G., Gitay-Goren, H., and Keshet, E. 1993 Patterns of expression of vascular endothelial growth factor (VEGF) and VEGF receptors in mice suggest a role in hormonally regulated angiogenesis. J. Clin. Invest. 91:2235-2243.

20. Neulen, J., et al. 1998. Secretion of vascular endothelial growth factor/vascular permeability factor from human luteinized granulosa cells is human chorionic gonadotrophin dependent. Mol. Hum. Reprod. 4:203-206.

21. Ferrara, N., and Davis-Smyth, T. 1997. The biology of vascular endothelial growth factor. Endocr. Rev. 18:4-25.

22. Thurston, G., et al. 1999. Leakage-resistant blood vessels in mice transgenically overexpressing angiopoietin-1. Science. 286:2511-2514.

23. Thurston, G., et al. 2000 . Angiopoietin-1 protects the adult vasculature against plasma leakage. Nat. Med. 6:460-463.

24. LeCouter, J., et al. 2001. Identification of an angiogenic mitogen selective for endocrine gland endothelium. Nature. 412:877-884.

25. McConnell, N.A., et al. 2002. Water permeability of an ovarian antral follicle is predominantly transcellular and mediated by aquaporins. Endocrinology. 143:2905-2912.

26. Palumbo, A., and Yeh, J. 1994. In situ localization of apoptosis in the rat ovary during follicular atresia. Biol. Reprod. 51:888-895.

27. Hazzard, T.M., and Stouffer, R.L. 2000. Angiogenesis in ovarian follicular and luteal development. Baillieres Best Pract. Res. Clin. Obstet. Gynaecol. 14:883-900. 